\title{
Evolution of Brahui Journalism
}

\author{
Muhammad Yousaf \\ M.Phil Scholar in Brahui Department \\ Dr. Liaqat Ali Sani \\ Associate Professor Department of Brahui \\ University of Balochistan Quetta
}

\begin{abstract}
This research article mirrors evolution of Brahui journalism in form of Weekly newspapers, monthly magazines, Bi-monthly reports and quarterly published literary reports. Some contributions, related to the journalism in Balochistan and Brahui journalism, have been researched, such as a brief history of Brahui journalism by AfzalMurd, 'Contribution of Magazines \& Journals for the Promotion of Brahui Language \& Literature' by Haji Abdul Lateef Bangulzai, and "Balochistan Mein Ablagh-e-Aama, Aghaz o Irtaqa” by professor Seemi Naghman Tahir. These are marked as appreciative initiatives; however, no researches have been carried out in the past to document the history and evaluation of print and electronic media in Brahui language. The comparative and analytical approach of study has been adopted in this article and has used secondary sources for conduct of this study.
\end{abstract}

Keywords: Journalism, Publication, Brahui language, launch, Magazine, Newspaper editor, editorship 


\section{Introduction:}

Brahui language is second widely spoken language of Baloch in Balochistan. Apart from Balochistan, the speakers of Brahui language inhabit in Afghanistan, Iran and Turkmenistan. There are a large number of Brahui speaking people in districts of Kalat, Mastung, Khuzdar, Lasbela, Nushki, Panjgure, Hub, and Jackababad, including Shikarpur, ShedaadKoat and Karachi of Sindh province in Pakistan. This gives birth to a as a well-established fact that, the print media has a great role in promotion of a language and literature. Though, Brahui journalism could not advance professionally, the role of Brahui magazines, weekly newspapers and quarterly published reports cannot be ignored in promoting its literature of the language.

The birth of Brahui journalism formally began in 1923, with the publication of Monthly "Al-Haq". Further, another Monthly "Muallim Sariab" published in 1950 from Quetta, in Brahui, Balochi, Pashtu and Persian. Brahui language was also given a great part in Weekly Naw-eWatan in 1953 and Monthly Balochi Karachi in 1956. In 1960 the history of Brahui literature and journalism witnessed the launch of Weekly Eilum Mastung, which is still being published. There are a large number of monthly magazines, weekly newspapers, annual and bi-annual reports including radio and television those have contributed in the growth of Brahui journalism.

\section{Brahui Print Media from 1923 to 1956}

\section{Monthly AlHaq}

The birth of Brahui journalism properly begins in 1923, with the publication of Monthly "Al-Haq" DeenPorKanGarah. Maulana 
Muhammad Umar Deenpori was founder of this monthly magazine and as such this is regarded as the first magazine ever published in the Brahui language. The magazine published religious articles aimed at sensitizing the women over religious matters in the face of Christan missionary crusade in Balochistan Monthly "Al-Haq also was published in the editorship of two females namely Ume Shareef and Ume Hanif (Murad Khaton and HazarNaz), who were the wives of Maulana Muhammad Umar Deenpori. Monthly Al-Haq is said to have been published from 1923 to 1930, however, this cannot be justified due to the unavailability of any issues of the magazines in archival records.

\section{Monthly "Mohalim" Sariyab}

Maulana Abdul Baqi Durkhani launched Monthly "Mohalim" Sariyab in 1950. Monthly “ Mohalim” Sariyab was published in Urdu but Brahui and Balochi write-ups were given special priority for publication. National and international news were covered with a great interest. Prominent Baloch journalist Abdul Rehman Ghaoor also worked as editor at the Monthly "Mohalim" Sariyab. The imposition of Press Ordinance by the then dictator Ayub Khan caused shutdown of this magazine in 1961.

\section{Monthly "Mubaligh" Mastung}

Maulana Abdul Ghafoor Durkhani launched Monthly "Mubaligh" Mastung in 1950; it would publish in Urdu but gave special placement to Brahui writings. Due to the financial crisis its printing process was closed in 1961. It reappeared as Weekly newspaper in 1980; however, it could not publish constantly and stopped its publication in 1990 .

\section{Weekly "Naw-e- Watan}

Ghulam Muhammad Shahwani launched Weekly "Naw-e- Watan in 1953. In a short time this weekly paper gained a great recognition 
among people in Balocistan. Weekly "Naw-e- Watan published in Urdu but it published Balochi and Brahui write-ups as well as it introduced investigative journalism in Balochistan. Weekly "Naw-e- Watan was banned in June 1955 against criticism on government through anonymous letter by a female to the editor.

\section{Naw-e-Bolan Quetta}

In 1956 Abdul Rehman Kurd launched Bi-monthly Naw-e-Bolan in Urdu from Mastung and later from Quetta. It also published writings in Brahui language on literature, culture and history. Naw-e-Bolan Quetta was banned in 1958 and later in 1960, till 1989 this paper published regularly. Since 2005 it is being publish as a magazine.

Mualan Muhammad Umar DeenPori, Ghulam Muhammad Shahwnai, Maulana Abdul GhafoorDurkhani, AbdulbaqiDurkhani, Abdul Rehamn Ghaoor and Abdul Rehman Kurd are the founding journalists in Brahui language who worked for promotion of Brahui journalism and literature.

\section{Brahui journalism from 1960 to 2000}

\section{Weekly Eilum Mastung}

Noor Muhammad Parwana launched first weekly newspaper of Brahui language on 24 February 1960. Initially it published Bi-monthly but soon it was published as weekly newspaper. The medium of newspaper is Brahui language but it also publishes news and opinions in Urdu. Since its launch Weekly "Elum" Mastung has introduced a large number of writers and poets in Brahui language. Elum is one of the regular newspapers of Brahui language.

\section{Monthly Olos Balochi Quetta}

The Tribal Publicity Department of Ministry of Information commenced publication of Monthly "Olous" in December 1991. Monthly 
Olous Balochi Quetta published in Balochi but some pages in the magazine were also fixed for Brahui contribution. This magazine often published till 1991. Amanullah Gichki, Kareem Dashti, Abdul Ghaffar Nadeem, Malik Toti Khan, Sorat Khan Marri, Abdul Qadir Aseer Shahwani and Abdul Razaq Sabir one after the other remained editor of Monthly Olos Balochi Quetta.

\section{Monthly Balochi Karachi/Quetta}

Abdul Wahid Azat launched Monthly Balochi Karachi/Quetta in 1956 from Karachi. It could not be published after two years; however, it commenced republishing in 1978 till the death of Abdul Wahid AzatJamaldini. Monthly Balochi published Brahui contributions of prose and poetry. From 1986 it is being published in editorship of Abdul Wahid Bandeeg.

\section{Monthly "Awal" Khuzdar}

The Tribal Publicity Department of Ministry of Information commenced publication of Monthly "Awal” Khuzdar in September 1975. It remained a popular magazine of Brahui language as prominent writers contributed in it. After 1979 it could not publish regularly, however, it played a great part by publishing Brahui literature.

\section{Weekly Khana Badoosh Mastung}

Weekly "KhanaBadoosh" Mastung is being published under the editorship of Deen Muhammad Shahwani in Urdu and Brahui.

\section{Monthly Alqalam Mastung}

Malik Fiyaz Ali launched Monthly Alqalam Mastung in July 1989. Monthly "Alqalam" Mastung published in Urdu but it also placed Brahui write ups. It encouraged young and new writers; it also published contributions of prominent writers of Brahui language writers. 


\section{Monthly Keher Tahar}

Dr.Dad Muhammad Khadim, published Keher Tahar newspaper from Jacobabad in 1990. It publishes in Sindhi, however, among the four pages one of them is set for Brahui language.

\section{"Subha Nau Chaghi"}

Abdul Haq Samlzai is publishing "Subha Nau Chaghi" as editor since May 1990 in Urdu Brahui and Balochi.

\section{Monthly Tawar Mastung}

Khadim Lehri commenced publishing Monthly Tawar from Mastung in Brahui language in 1992. After more than a decade it was converted in a daily newspaper. Currently, it is only available online but publishes a weekly page in Brahui language.

\section{Monthly Aadeenk Mastung}

Yousaf Hassan Yousaf launched monthly “Aadeenk” Mastung in May 1996, where Yousaf Hassan Yousafserved as editor and Hameed Aziz Abadi worked as Sub-editor. Aadeenk published in Urdu and Brahui.

\section{Monthly Harboi Kalat}

Yousaf Ajab Baloch launched Monthly Harboi in October 1999. It Published in Brahui language. Yousaf Ajab worked as chief editor of the magazine and Shakir Khalil served as editor. Later Allah Buxlehri as editor and Mehboob Shahwani joined it as sub-editor. Monthly Harboi could not continue its publication due to the financial crisis. However, once again it is being published by Muhammad Yousaf Zehri regularly.

\section{Monthly Girooshk}

Montly Girooshk has credit to be the first Brahui magazine for children. Its first edition was published in December 1999 and second one 
in January 2000. Young writer Noor Pirkani was its Chief Editor and Imdad Ali Mengal was affiliated as editor. As like other magazines of Brahui language the "Girooshk" team could not continue its publication. Sangat Quetta, Gindar Kalat, Sangat Kalat and some others published bilingual or in Brahui language but their printing process could not be maintained.

\section{Brahui Journalism from 2000 to 2016}

\section{Monthly Istar Surab}

Istar emerged as a monthly Magazine in January 2001 from Surab district of Kalat, under the editorship of Illahi Bux Anjum Brahui. Monthly "Istar" Surab owns credit of being published since its publication. It publishes in Brahui language, focusing on Brahui literature and language. Currently, Rehmatullah Zahid is leading the "Istar" team as editor.

\section{Monthly Hatam Kalat}

The editor and founder of monthly Hatam Kalat, Shaheen Baranzai published and released its first copy in 2003. It published in Brahui language. However, publication of "Hatam" could not sustain and it vanished from market in 2016.

\section{Weekly Talar Noshki}

Weekly Talar is being published from Noshki since 2004. Abdul Raziq Ababaki is its founder and editor. It is one of the most circulated newspapers of Brahui language with its unique write ups, special editions and editorials and editorial policy. It has encouraged and introduced dozens of writers since its launch. The online edition of Talar is also available for the global audiences.

\section{Daily Talar Noshki}


Daily Talar Noshki is the only Brahui language newspaper being published in Balochistan. It is being published since July 2005. Abdul Raziq Ababaki is its founder and editor. Daily Talar Noshki covers national and international news for Brahui readers. As well as it publishes Brahui literature and current affairs. The online edition of Talar is also available for the global audiences.

\section{Monthly Meher Noshki}

Monthly Meher commenced its publication in 2005 from Noshki. It is published in Brahui language but also covers write up in Urdu and English about Brahui language. Muhammad Noor is current editor of Monthly "Meher" Noshki.

\section{Monthly Aasyab Noshki}

Nasir Rahi launched first copy of Monthly Aasyab in 2005 in Urdu and Brahui from Noshki.It covers national and international issue in Brahui and Urdu literature in the mentioned languages.

\section{Weekly Parave Kalat}

Weekly Parave Kalat is being published since 2010, under the editorship of Saeed Noor. It regularly publishes in Brahui language. It is also available for the global audience on social media.

\section{Bi-monthly Kokar}

Young journalist "Kokar" Ahmed Naheem is editor of "Kokar". It is being published Bi-monthly in Brahui language since January 2010.

\section{Quarterly published Magazines and Literary reports}

Different Literary groups and societies have published their quarterly magazines and reports, in which some of the well known ones are Day Tik, Sangat, Godi, Kappot, Talar, Chiltan and Chiragh.

\section{Quarterly Sangat}


Brahui poet and writer Johar Brahui published Quarterly "Sangat" from Fareedabad Sindh in 1981.Sofar, 20 editions of Sangat have been published.

\section{Quarterly Day-Tik}

Brahui Adabi Society started publication of Day-Tik in 1986, initially it published monthly, and however, later it was converted in a quarterly magazine. It has also published special editions about famous Brahui poets, and intellectuals such as Ghulam Nabi Rahi, Nadir Qambrani, Abdurrahman Kurd, Peer Muhammad Zubairani, JabbarYar and Noor Muhammad Parwana.

\section{Goodi, Hyderabad}

Ali Ahmed Shad starred publication of Goodi, Hyderabad, it was a series of literary reports in 1986. It could only print its two editions of "Sakina" number and "Aman" number.

\section{Bi-annual Kapoot Jacobabad}

Azizullah Aziz, Dad Muhammad Khadim and some other Brahui writers launched publication of "Kapoot" a bi-annual magazine in 1984. It could only publish its three editions.

\section{Talar}

Talar was an effort of Muhammad Yousaf Maooj, who launched under the influence of Brahui Adabi Tanzeem in 1984. It was a digest sized magazine. It lasted up to the release of a dozen editions.

\section{Chiltan Mastung}

Chiltan Mastung Published under Chiltan Adabi Dewan Mastung. Malik Ali Murad Khaki, Malik Fiyaz AliBaloch began its release in 1987. Quarterly Chirag Noshki 
Quarterly "Chirag" Noshki published from Noshki in 2005 and 2006 in Balochi, Brahui, Urdu, Pashtu and English languages. Agha Zahid Hussain led Chirag team as Chief Editor, whereas Malik Akram Mengal worked as editor and Ghum khawar Hayat compiled and edited Brahui prose and poetry.

Some organizational magazines mainly different factions of Baloch Students Organization (BSO) have published Brahui literature and their political write-ups in Brahui language. The magazines and their organizational reports include Sang, Pajar, Girook and Baam.

Apart from the, abovementioned newspapers and magazines, Brahui language and literature have been given priority to have the great place in Weekly "Chung” Quetta, Weekly Shaal Quetta Aljzeera Quetta, Bazam Balochistan, Monthly Tehreek, Monthly Taqdeer,Mobthly Monthly Chatan, Chiltan, Sahar, Shama Adab, Monthly Sangat, Sada-eAmrooz, Monthly Sada-e-Haq, Sareer Bolan, Qalam Qabila, Monthly Mehwar, Nashnas, Nokeen Daur, Wadi, Daily Awam, Monthly Jabal, Monthly Tohbay, Monthly Branz, Sarawan, and Monthly Watan. Pakistan

Television and Radio, weekly and monthly columns in daily and monthly newspapers have been a great source of information promotion for Brahui journalism and literature.

\section{Conclusion:}

Similar to other languages in Pakistan, Brahui language has its own journalism history. Since its birth dozens of monthly magazines, weekly newspapers, literary reports, a daily newspaper, radio and state owned Pakistan Television (PTV) have played a great role to promote Brahui poetry and prose. Besides literature, national and international issues have also been covered and published in Brahui language (Sani: 2015:101) 
However, due to the financial crises many printing organizations could not sustain and they failed to serve Brahui language.

\section{Reference:}

Ahmed, Kamal-ud-din, (1978) Sahafat Waddi Bolan Mehn, Balochi Academy.

Naghman, Seemi Tahir, Professor (19....)“Balochistan Mein Ablagh-e-

Aama, Aghaz o Irtaqa"

Murad Afzal, (2011) Nidarah.

Bangulzai, Abdul Lateef, (2014) "Contributions of Magazines \& Journalism for the Promotion of Brahui Language \& Literature"

Bi-monthly Kukar January 2010.

Bedar, Abdul Qayum (2014) “Neyari ta Adab” (3-10)

Chiltan, Mastung, Chiltan Adabi dewan, November 1987.

Goodi, Hyadarabad, 1986,

Kurd, Dr Saleem (2015) “ Naw-e- Bolan

Kapoot, Hatam, 1984.

Lehri, Abdul Bari (2015)“Maktaba Durkhani ki Ellmi wa Deeni Khidmat ka Jahiza" (139-140)

Mansoor, Haji Abdul Hyath,( 2009) “ Baba-e-Brahui wa Eilum

Monthly Awal Khuzdar, June 1970

Monthly Harboi Kalat October 1999. 
Monthly Adeenk Mastung May 1996.

Monthly Balochi Karachi/ Quetta.

Monthly Hatam Kalat, October 2003.

Monthly Istar Surab, January 2001.

Monthly Meher Noshki, January 2005.

Monthly Girooshk, December 1999.

Quarterly Chirag Noshki, January, February, March 2006.

Quarterly Day Tik Quetta, April, June 1990.

Quarterly SangatBrahui Publication Fareedabad, Sindh, Ghazi, 1981.

Saif, Haji Saifullah (August 2014) Monthly Istar' Surab.

Weekly Talar, Noshki, January 2004. 